relatedness with CS words, a difference score was computed for each experimental word. The difference score for a given experimental word was found by subtracting the mean confidence rating for its control counterpart (i.e., the control word matched for frequency, etc.) from the word's own mean confidence rating. The correlation between these difference scores and degree of associative relatedness approached statistical significance, $\operatorname{rho}(16)=.44, \mathrm{p}<.10$. While moderate, the correlation is nevertheless impressive in view of the modest range of variation in degree of associative relatedness between experimental and CS words.

In general, the present results favor the IAR hypothesis. That is, the presence of associative relatedness alone is seemingly sufficient cause for generating the false-recognition effect. However, the possibility remains that associatively related words that are neither synonyms nor antonyms may, nevertheless, be related semantically via other, unmeasured, shared semantic features. Analyses of these semantic features, and the determination of their role in mediating the false-recognition effect, remain problems for future research.

\section{REFERENCES}

Anisfeld, M. \& Knapp, M. E. Association, synonymity, and directionality in false recognition. Journal of Experimental Psychology, 1968,77, 171-179.

Cramer, P. Evidence for a developmental shift in the basis for memory organization. Paper presented at the annual meeting, Psy chonomic Society, St. Louis, November 1972.

Fillenbaum, S. Words as feature complexes: False recognition of antonyms and synonyms. Journal of Experimental Psychology, 1969, 82, 400-402.

Grossman, L., \& Eagle, M. Synonymity, antonymity, and association in false recognition. Journal of Experimental Psychology, 1970, 83, 244-248.

Hall, J. W. Effects of IAR occurrence during learning on confidence in judgments during recognition. Journal of Experimental Psychology, 1969, 79, 578-580.

Underwood, B. J. False recognition produced by implicit verbal responses. Journal of Experimental Psychology, 1965, 70, 122-129.

\section{NOTE}

1. We are grateful to Grossman and Eagle for making their word lists available to us.

(Received for publication May 25, 1973.)

\title{
The influence of time course variables on REM sleep
}

\author{
H. W. AGNEW, JR., and W. B. WEBB \\ University of Florida, Gainesville, Fla. 32601
}

This paper studies the effect of age, length of prior wakefulness, length of sleep, and a circadian influence on REM sleep. Age is a major determinant of REM amount up to the early teens, but has little effect thereafter. Length of prior wakefulness has little or no effect on REM amount. Length of sleep has a major influence on REM amount, with longer sleep times producing greater amounts of this type of sleep. There is a strong circadian effect on REM, with early night sleep periods producing little REM and early morning periods a relatively great amount of REM.

In an earlier report (Webb, Agnew, 1972), we reviewed and analyzed the responsiveness of Stage 4 sleep (deep sleep) to changes in four variables: age, length of the sleep period, length of prior wakefulness, and the onset time of the sleep period (a circadian effect). Because our data were drawn from studies not originally designed specifically to study these influences, the data on prior wakefulness was confined to the first $3 \mathrm{~h}$ of sleep and the data on circadian effects were limited in onset points. Nevertheless, since Stage 4 sleep is generally distributed within the first $3 \mathrm{~h}$ of sleep we felt confident of our generalizations.
This paper reviews the same data relative to changes in four variables and REM sleep. Stage REM is associated with rapid eye movements and a Stage 1 electroencephalogram (EEG). This stage of sleep was first recognized by Aserensky \& Kleitman (1953) and is associated with visual dreaming in humans (Dement \& Kleitman, 1957). There are numerous reports that: when Ss are deprived of REM sleep they tend to recover it on subsequent nights in a manner which would suggest that it represents a basic need state (Dement, 1960). Since, as will be noted below, this stage of sleep is predominant after the first $3 \mathrm{~h}$ of sleep, the data relative to prior wakefulness is severely limited. However, it is likely to be some time before the needed data on age and length of sleep are available, but the nature of the influence of prior wakefulness on REM in the first $3 \mathrm{~h}$ of sleep show interesting relations to Stage 4 , and we feel that this limited interim report is appropriate.

The data for the present report was gleaned over the course of 10 years' research on human sleep patterns. Our procedures for obtaining EEG sleep records and for sleep stage scoring are described in a comprehensive report (Agnew \& Webb, 1972). Essentially, a 1-min epoch of EEG is scored as Stage REM if rapid eye movements occur contiguously with a Stage 1 EEG. The 


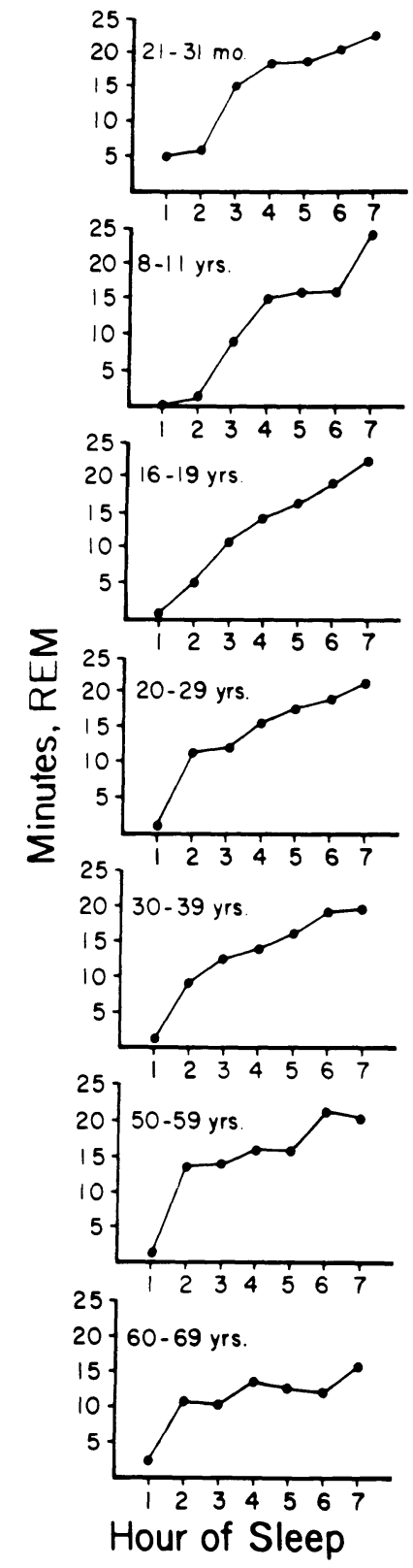

Fig. 1. Min of REM sleep found in each hour of sleep for seven age groups.

data for the present report are based on a number of studies of the normative characteristics of sleep of Ss aged 21 months to 69 years. Most of these data have been published in studies of single age groups (Williams, Agnew, \& Webb, 1964: Kohler, Coddington, \& Agnew, 1968; Ross, Agnew, Williams, \& Webb, 1968; Agnew, Webb, \& Williams, 1967). The data on prior wakefulness and on the circadian effect were developed from a series of studies on variations of sleep patterns within the 24-h cycle (Webb. Agnew, \& Williams, 1971: Webb \& Agnew, 1971).

The effect of sleep length on REM amount was initially suggested by a normative study of sleep in the young adult male (Williams, Agnew, \& Webb. 1964). In this study. we reported that $53 \%$ of the total REM amount occurred after the fifth hour of sleep. Later. in a study which restricted sleep to $3 \mathrm{~h}$ in 24 (Webb \& Agnew, 1965), the REM amount was sharply reduced: and, on recovery nights (10.14 h long), $69 \%$ of the total REM occurred after the sixth hour of sleep. Independent support for our suspicions came from a study of extended sleep by Verdon (1968).

Figure 1 show's the relation between sleep length and REM amount in nighttime sleep periods $7.8 \mathrm{~h}$ in length. Presented is an hour-by-hour analysis of the amount of REM in the sleep of six age groups. These curves reflect several complex and interacting features of REM sleep. REM sleep occurs in relatively short bursts, which vary from night to night in terms of their number, onset time. length, and interval between episodes. In 72 nights of sleep of the 20-29-year-olds, every night contained at least three episodes of REM sleep. Fifty-eight nights contained a fourth REM period and 21 nights a fifth REM period. There were no occasions of a sixth REM period in these 7-8-h sleep records. The mean onset time of the first REM period was $99 \mathrm{~min}$, but, Fig. 2 shows the distribution of REM onsets about this mean to be quite variable. Only $24 \%$ of the REM onsets occurred within $10 \mathrm{~min}$ of $99 \mathrm{~min}$. Subsequent REM periods occurred at a mean of 196, 289, 367, and 409 min after the onset of the first Stage 1. From the distributions for successive REM periods, it can be seen that as the number of episodes increase, the interval between them decreases. Finally, in general, as the number of episodes increase, so do their lengths. In the young adult data, the first four REM periods were successively $20,26,35$. and $36 \mathrm{~min}$ in length.

Figure 3 shows that REM time is strongly influenced by the age of the $S$ in the first decade of life, but thereafter the amount of REM tends to level off and remain constant as $\mathrm{S}$ age is increased. Similar results have been reported by others (Roffwarg et al, 1966).

Analysis of the data for the effect of prior wakefulness on REM amount reveals only a limited

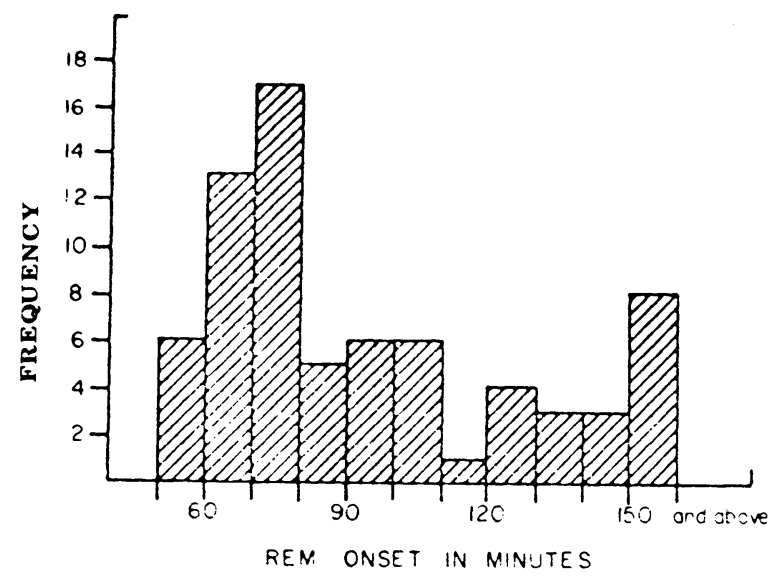

Fig. 2. Frequency distribution of REM onsets after the onset of the first stage 1 of sleep. 
relationship. This is shown in Fig. 4. It may be noted. additionally. that Ss sleep-deprived do not, subsequently, show more REM sleep unless they are permitted to sleep for a longer than normal length of time (Berger \& Oswald, 1962).

The first clues to a circadian influence on REM sleep came from a study of daytime naps (Webb, Agnew, \& Sternthal, 1967). Ss sleeping at 9 a.m. obtained $32 \mathrm{~min}$ of REM in 2 h, but sleep at 12:30 p.m. yielded only $12 \mathrm{~min}$ of REM and sleep at 4 p.m., $8 \mathrm{~min}$. The clear influence of a circadian variable could not be stated in this paper, since time of the sleep period was confounded with length of prior wakefulness. In a later study (Webb \& Agnew, 1971), length of prior wakefulness was held constant. In this study, sleep periods between 11 p.m. and 3 a.m. produced $39 \mathrm{~min}$ of REM sleep, while sleep periods between 3 a.m. and 7 a.m. produced $84 \mathrm{~min}$ of REM sleep. In the early morning sleep period, REM latency is decreased and the temporal ordering of REM within the sleep period is a much flatter curve than that shown in Fig. 1.

REM sleep and Stage 4 sleep have been shown to be systematically responsive to four variables that are the common surrounds of the normal sleep process: age, prior wakefulness, sleep length, and time of sleep onset. In combination, specification of these variables permits a remarkably accurate prediction of the amount of each stage that will be present during sleep. Since these stages constitute between $40 \%$ and $50 \%$ of the sleep process and are totally different in nature, the results are impressive.

Of particular interest is the difference in the relationships found: (1) Age is a primary determinant of REM amount up to the early teens, whereas Stage 4 begins to diminish beyond the age of 30. (2) Prior

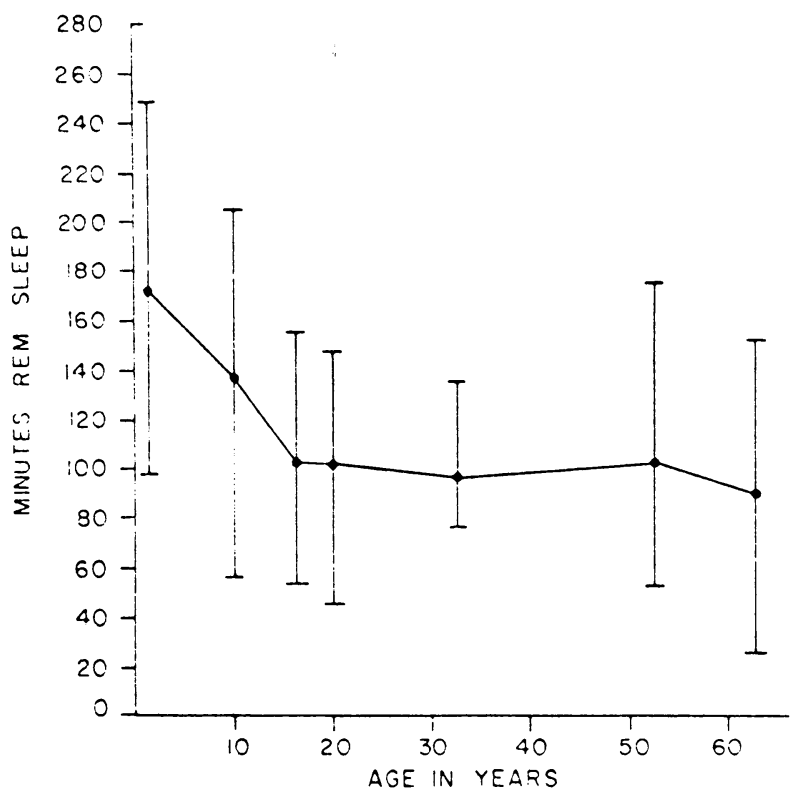

Fig. 3. Min of REM found in the sleep of seven different age groupings of Ss.

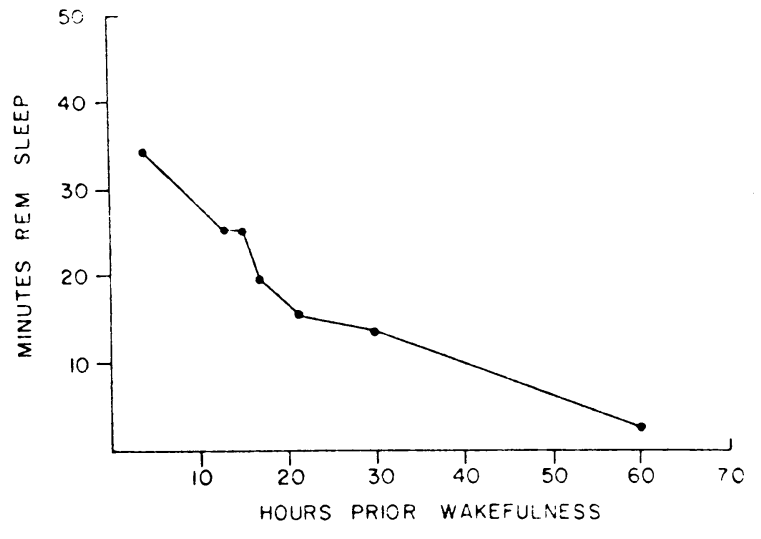

Fig. 4. Relationship between length of wakefulness prior to sleep and amount of REM in first $3 \mathrm{~h}$ of sleep.

wakefulness is a strong determinant of Stage 4 sleep, but a limited influence on REM sleep. (3) Onset time or circadian effects show a strong relationship to REM and less so with Stage 4. (4) Stage 4 is more prominent in the early part of a sleep period, and REM in the latter part. These different functional relations indicate different processes and are supportive of the differentiation of the two stages at biochemical and neurophysiological levels.

\section{REFERENCES}

Agnew, H. W., Jr., Webb, W. B., \& Williams, R. L. Sleep patterns in late middle age males: An EEG study. Electroencephalography \& Clinical Neurophysiology, 1967 , $23,168-171$

Aserinsky, E., \& Kleitman, N. Regularly ocurring periods of eye motility and concomittant phenomena during sleep. Science $1953,118,273-274$.

Berger, R. J., \& Oswald, I. Effects of sleep deprivation on behavior, subsequent sleep, and dreaming. Journal of Mental Science, 1962, 108, 457-465.

Dement, $W$. C. The effect of dream deprivation. Science, 1960 , 131, 1705-1707.

Dement. W. C., \& Kleitman, N. Cyclic variations in EEG during sleep and their relations to eye movements, body motility, and dreaming. Electroencephalography \& Clinical Neurophysiology, 1957, 9, 673-690.

Kohler, W. C., Coddington, R. D., \& Agnew, H. W., Jr. Sleep patterns in 2-year-old children. Journal of Pediatrics, 1968, 72, 228-233.

Roffwarg, H. P., Muzio, J. N., \& Dement, W. C. Ontogenetic development of human sleep-dream cycle. Science, 1966, 152, 604-619.

Ross, J. J., Agnew, H. W., Jr., Williams, R. L., \& Webb, W. B. Sleep patterns in pre-adolescent children: An EEG-EOG study. Pediatrics, $1968,42,324-335$.

Verdon, P. Sleep satiation: Extended sleep in normal subjects. Electroencephalography \& Clinical Neurophysiology, 1968, $24,417-423$.

Webb, W. B., \& Agnew, H. W., Jr. Sleep: Effects of a restricted regime. Science, $1965,150,1745-1747$.

Webb. W. B. \& Agnew, H. W. Jr. Variables associated with split-period sleep regimes. Aerospace Medicine, 1971, 42, 847-850.

Webb, W. B., \& Agnew, H. W., Jr. Stage 4 sleep: Influence of time course variables. Science, 1972, 174, 1354-1356.

Webb, W. B.. Agnew, H. W., Jr., \& Sternthal, H. Sleep during the early morning. Psychonomic Science, 1966, 6, 277-278.

Webb, W. B., Agnew, H. W., Jr., \& Williams, R. L. Effect on sleep of a sleep period time displacement. A erospace Medicine. $1971,42,152-155$.

Williams, R. L. Agnew, H. W. Jr. \& Webb, W. B. Sleep patterns in young adults: An EEG study. Electroencephalography \& Clinical Neuorphysiology, 1964, 17, 376-381.

(Received for publication Mạ 27. 1973.) 